\title{
Partial Reflection of Hyaluronan Molecules Inside the Taylor-cone During Electrospray
}

\author{
Zhikai Tan \\ School of Biology, Hunan University, Changsha, Hunan, China
}

Received 2012

\begin{abstract}
Electrospray (ES) is of great interest in research for its finely controlled delivery of molecules. This study investigated mechanisms involved in the electrospray of biological macromolecules which may cause spraying instabilities. Hyaluronan (HA) has been studied for its biological significance. A mixture of ethylene glycol and deionized water with volume ratio of 1:1 is used to dissolve HA molecules. HA solutions with different concentrations and molecular sizes are investigated. Experimental results demonstrate that the molecular size and solution concentration of macro-molecules are critical factors affecting the spraying process. A concentration reduction of HA molecules happened during the ES process, and a hypothesis on partial reflection of HA molecules inside the Taylor-cone is presented in the study.
\end{abstract}

Keywords: Electrospray; Macro-molecules; Reflection; Talor-cone

\section{Introduction}

With the emergence of nanotechnology, researchers become more interested in the electrospray (ES) technique in recent years due to its versatility and potential for applications in diverse fields [1,2]. By applying a suitable voltage to a conducting liquid supplied into a capillary, the liquid meniscus will take a form of cone $[3,4]$. Further increasing the electric field on the surface of the liquid to overcome the surface tension, a controlled $\mu \mathrm{m}$-sized jet will emerge from the tip of the liquid cone which is called the stable cone-jet mode [3]. Consequently, by manipulating the microscopic fluid jet and ejected charged droplets onto a desired place, an e-jet delivery or deposition can be achieved. By using micro-sized fine nozzles, ES can operate at a low flow rate, which accordingly makes the delivery of tiny volumes possible [5].

The electrospray of macromolecules has seen a tremendous increase in research and commercial attention over the past decade due to a number of applications such as in fabrication of enzyme electrodes [6], micro-cells for biochemical reactions [7, 8] and thin polymer films [9]. In this study, molecules of Hyaluronan (HA, i.e. hyaluronic acid) are chosen to be investigated due to its unique rheological properties and complete biocompatibility. As a naturally occurring linear polysaccharide, HA is popularly found in connective tissues such as umbilical cord, synovial fluid and vitreous etc. Meanwhile, HA has been widely used in fields of drug delivery, cell encapsulation and tissue regeneration [10]. The chemical structure of HA consists of alternating disaccharide units of D-glucuronic acid and $\mathrm{N}$-acetyl-D-glucosamine. Molecular weight of HA can vary from $5 \mathrm{kDa}$ to $2.1 \mathrm{MDa}$ in vivo which will result in varied molecular sizes [11].

Increasingly considerable attentions has been received nowadays for the electrospray of HA solutions into types of microfibers or membranes [10,12]. However, how to achieve stable electrospray of HA solutions still remains problematic due to uncertain reasons. Fluid motions within the Taylor-cone and the way that carries HA macro-molecules is of some relevance in understanding of HA spraying jet stability controlling difficulties. These motions during electrospray are driven by the tangential electrical stress acting on the liquid-gas interface and the flow rate injected through the electrified emitter [13].

Based on work of Hayati, Barrero and Sheldon for liquid motions inside the Taylor-cone containing both experimental results and theoretical modelling, there is a recirculating meridional motion during the ES process, liquid moves towards the apex along the generatrix and away from it along the axis as shown in Figure 1 [13-16],. Their results show that only fluid particles lying close to the surface are ejected through the jet while the rest recirculates towards the apex along the generatrix and away from it along the axis [13,17]. When solutions of HA macro-molecules are sprayed under stable cone-jet conditions, the anticipated molecules movement could be as shown in Figure 1. HA molecules move following liquid towards the apex and away from it along the axis $[15,16]$.

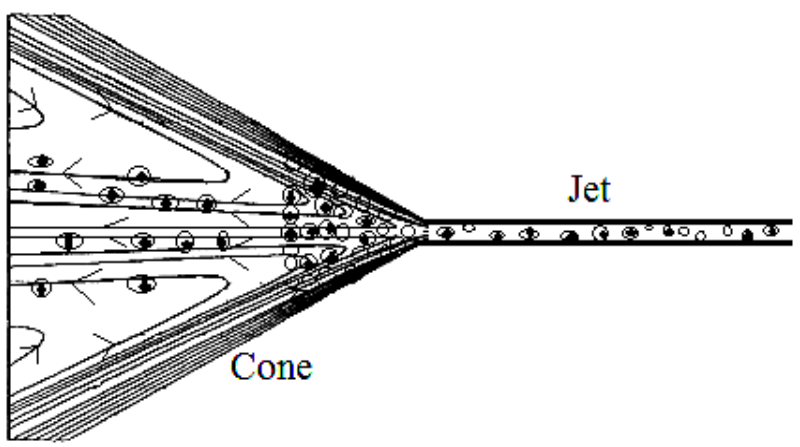

Figure 1. Streamlines and movements of HA molecules within the Taylor-cone during the electrospray: Meridional circulation of liquid with molecules towards the apex along the generatrix and away from the apex along the axis. 
During the process, only that fraction of HA molecules lying close to the surface are ejected through the jet while the rest recirculated towards the apex away from it along the axis [13, 17]. This recirculation may cause a back diffusion movement of HA molecules, and with a higher HA concentration nears the tip which would result in partial reflection of HA molecules during the electrospray. It is possible then that this process may cause instabilities to the ES process and a concentration reduction of HA molecules after the electrospray process.

\section{Materials and Methods}

Hyaluronan powders (sodium salt, molecular weight: 2.1 MDa) were purchased from Genzemy Co. (USA) and diluted in ethylene glycol and water $\left(\mathrm{EG} / \mathrm{H}_{2} \mathrm{O}\right.$, volume ratio=1) with different concentrations $(0.1,0.2$ and $0.4 \mathrm{mg} / \mathrm{ml})$. A sonication method was used to reduce the molecular size and chain length of HA [18]. After sonication, average molecular weights of HA were measured (200 kDa, $390 \mathrm{kDa}$ and $1000 \mathrm{kDa}$ ) by sizeexclusion high performance liquid chromatography (HPLC).

In order to examine the partial reflection of HA molecules, experiments were undertaken to investigate whether the electrospray process was acting as a filter of HA molecules or not. The hypothesis is that if the partial reflection takes place then downstream of the Taylor cone, liquid collect from the jet end may have a lower concentration of HA molecules than in the initial fluid solution. Figure 2 shows the experimental schematic of the ES apparatus for the study. The main components consist of fluid supply system, spraying nozzle, high voltage power supply, current measuring unit and an imaging system to monitor the spraying cone and jet.

Nozzles were made from glass tubes. A micropipette puller P-97 (Sutter Instrument Co.) was used to produce tips of nozzles down to $30 \mu \mathrm{m}$. Liquid contained in a small reservoir was forced through a narrow silica tube into the nozzle by pressurized nitrogen gas. All experiments were completed at atmospheric conditions at the emitter tip, with the same initial fixed pressure. The change in pressure as the reservoir drained was insignificant, as each spray used less than $0.5 \mathrm{ml}$, which resulted in a change in height less than $1 \mathrm{~mm}$.

The power supply (F.u.G. Electronik) can provide a voltage up to $6 \mathrm{kV}$. The spray distance between the nozzle tip and the

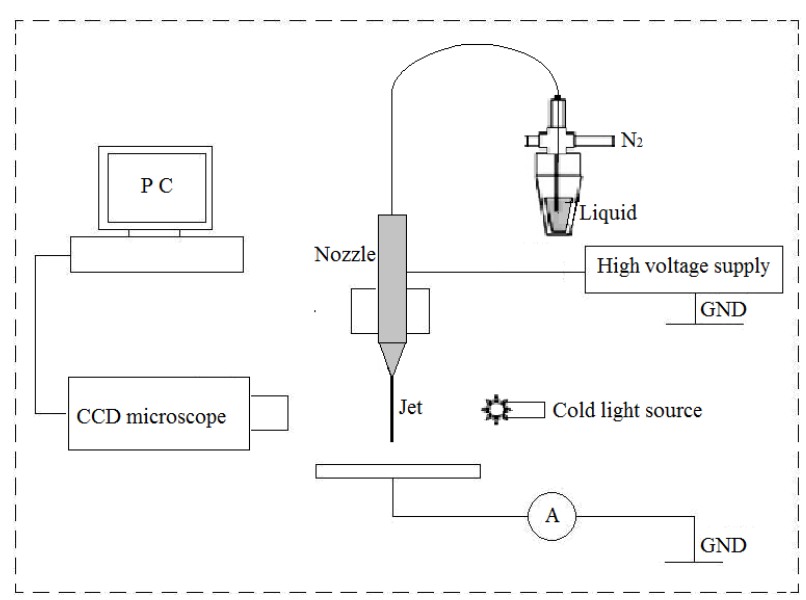

Figure 2. Experimental schematic for electrospray of HA solutions. substrate was set to $3 \mathrm{~mm}$. A voltage ranged in $2.3 \sim 2.5 \mathrm{kV}$ was applied to produce a stable cone-jet electrospray. The slight adjustment of the applied voltage was made to maintain a spraying due to different physical properties of solutions. The current was determined by measuring the voltage drop across a $100 \mathrm{M} \Omega$ resistor. An ISO-TECH IDM 207 voltmeter logged the voltage to a PC at a frequency of $2 \mathrm{~Hz}$.

The jet during an electrospray process was monitored by an optical system based on a microscope. Images were recorded and captured by a computer. The system consists of a combined zoom lens and a CCD camera (UEYE). An infinity corrected objective lens from Mitatoyo and a variable zoom from Thales Optem were used. The optical resolution can reach to $1 \mu \mathrm{m}$. Additionally, a cold light source was used for illumination.

\section{Results and Discussion}

Figure 3 shows optical micrographs of spraying pure EG/ $\mathrm{H}_{2} \mathrm{O}$ solvent and HA solutions (molecular weight: $1000 \mathrm{kDa}$ ) with concentrations of $0.1,0.2$ and $0.4 \mathrm{mg} / \mathrm{ml}$. Respectively, stable spraying jets were observed in HA solutions with concentrations of $0,0.1$, and $0.2 \mathrm{mg} / \mathrm{ml}$. However, further double the concentration up to $0.4 \mathrm{mg} / \mathrm{ml}$ the stable jet was not observed, which clearly indicated that the stable electrospray process can be hampered by adding HA macromolecules.

Varied concentrated HA solutions $\left(\mathrm{C}_{\mathrm{in}}\right)$ with different molecular sizes were electrosprayed at stable cone-jet mode for further investigations. Solutions of HA $1000 \mathrm{kDa}$ molecules were only be cone-jet electrosprayed when $C_{\text {in }}<0.4 \mathrm{mg} / \mathrm{ml}$ while other smaller HA molecules (200 kDa and $390 \mathrm{kDa}$ ) could reach the stable cone-jet electrospray at the highest concentration. Molecular size and concentration do affect the electrospray stability of HA solutions.

Samples of liquid from downstream spraying jet were collected every 20 minutes from the plate electrode after cone-jet electrospray have been reached, and then concentrations of samples $\left(\mathrm{C}_{\text {out }}\right)$ were measured. Concentration change $\mathrm{R}$ was calculated as following:
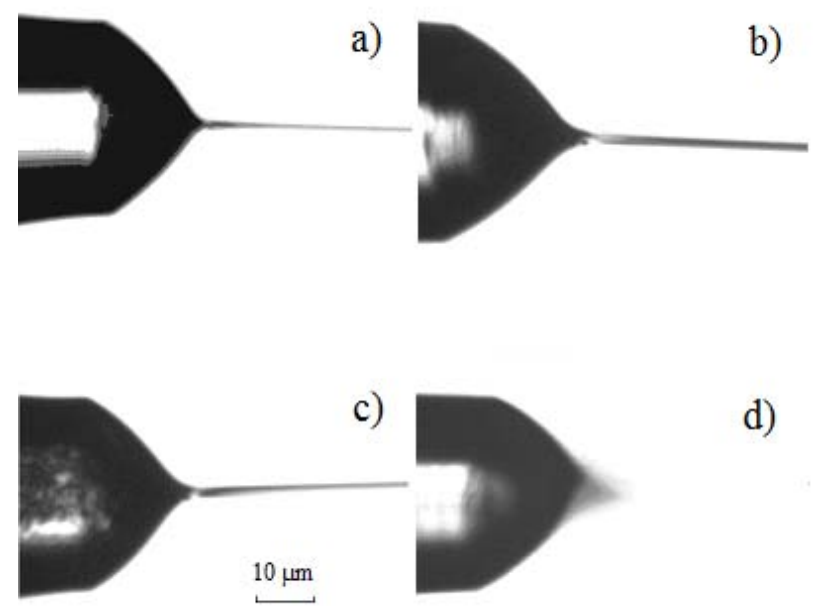

d)

Figure 3. Microphotography images showing the electrospray of solutions: a) electrospray of pure $\mathrm{EG} / \mathrm{H}_{2} \mathrm{O}$ solvent. b)-d) electrospray of HA (1000 kDa) solutions with a concentration of $0.1,0.2$ and $0.4 \mathrm{mg} / \mathrm{ml}$, respectively. 


$$
R=1-\frac{C_{\text {out }}}{C_{\text {in }}}
$$

Figure 4 gives results of HA concentration changes after 20 minutes cone-jet electrospray under varied initial concentrations. After electrospray, measured concentrations of HA have lower values, there is an obvious concentration reduction which is up to $30 \%$ (HA $1000 \mathrm{kDa}$ ) from Figure 4.

This is consistent with the hypothesis that a partial reflection of HA macro-molecules takes place during the cone-jet electrospray process. Figure $\mathbf{4}$ also indicates that varied molecular sizes of HA result in different concentration reduction values after spray at the same concentration, a larger HA molecular size will result in a greater reflection rate. The reduction value of the largest HA molecule (1000 $\mathrm{kDa})$ is almost three times greater than the one caused by the smallest molecule $(200 \mathrm{kDa})$ as listed in the figure. The size of HA molecules does affect the reflection process and a higher concentration would also slightly increase the concentration reduction value after ES for all HA molecules. Figure 5 shows that the reduction value decrease with spraying time, which indicated that the reflection of HA molecules is not constant during the electrospray.

Concentration reduction results of HA molecules after electrospray demonstrate the hypothesis that HA molecules create a higher concentration layer near the Taylor-cone tip which cause

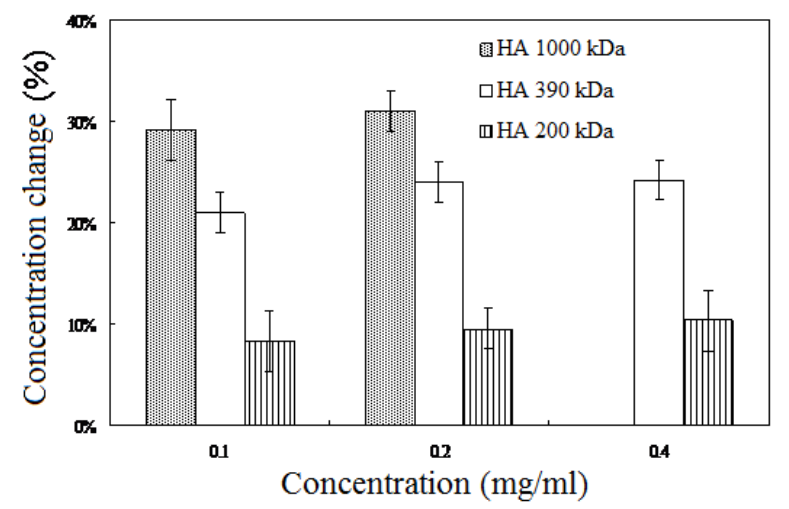

Figure 4. Concentration reductions of HA molecules after 20 minutes spray under different initial concentrations.

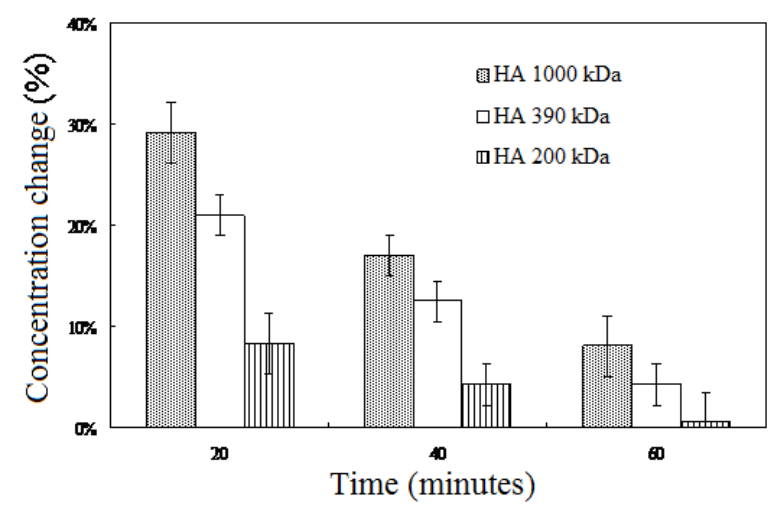

Figure 5. Concentration reductions of $\mathrm{HA}(0.1 \mathrm{mg} / \mathrm{ml})$ solutions after electrospray as a function of spraying time. molecules back diffusion during electrospray process. This HA partial reflection process is dependent of molecular sizes, solution concentrations and spraying time.

\section{Conclusions}

The electrospray of HA molecules is investigated in the study, and a partial reflection of HA molecules is found during the stable cone-jet electrospray. A hypothesis which describes the partial HA reflection is developed. It indicates the build-up of a concentrated HA layer near the Taylor-cone tip during electrospray and the development of HA concentration changes with spraying time. This work elucidates the mechanism may responsible for instability of electrospray process when using HA macro-molecules solutions. The concentration reduction during electrospray of macro-molecules has important implications for applications of electrospray. For example, this process may affect the signal when use ES for mass spectrometry, it can also affect in the fields of drug delivery, cell encapsulation and tissue engineering when using the ES technique.

\section{Acknowledgements}

The author acknowledges the financial support of Hunan University.

\section{REFERENCES}

[1] Oleg, V.S., “Tools of Nanotechnology: Electrospray”. Current Nanoscience, 2005, (1), 25-33.

[2] Park, J.-U., Lee, S., Unarunotai, S., Sun, Y., Dunham, S., Song, T., Ferreira, P., Alleyene, A., Paik, U., \& Rogers, J. "Nanoscale, Electrified Liquid Jets for High-Resolution Printing of Charge”. Nano Letters, 2010, (10), 584-591.

[3] Fernández de la Mora, J. “The Fluid Dynamics of Taylor Cones”. Annual Review of Fluid Mechanics, 2007, (39), 217-243.

[4] Fernández de la Mora, J., \& Loscertales, I.G. "The current emitted by highly conducting Taylor cones”. Journal of Fluid Mechanics, 1994, (260), 155-184.

[5] Wang, K., \& et al. "Fully voltage-controlled electrohydrodynamic jet printing of conductive silver tracks with a sub-100 $\mu \mathrm{m}$ linewidth”. Journal of applied physics, 2009, (106), 024907.

[6] Yogi, O., Kawakami, T., \& Mizuno, A. "Properties of droplet formation made by cone jet using a novel capillary with an external electrode”. Journal of Electrostatics, 2006, (64), 634-638.

[7] Delamarche, E., Juncker, D., \& Schmid, H. "Microfluidics for processing surfaces and miniaturizing biological assays”. Advanced Materials, 2005, (17), 2911-2933.

[8] He, M., Edgar, J.S., Jeffries, G.D.M., Lorenz, R.M., Shelby, J.P., \& Chiu, D.T. "Selective Encapsulation of Single Cells and Subcellular Organelles into Picoliter- and Femtoliter-Volume Droplets”. American Chemical Society Journals, 2005, (77), 1539-1544.

[9] Rietveld, I.B., Kobayashi, K., Yamada, H., \& Matsushigea, K. "Morphology control of poly(vinylidene fluoride) thin film made with electrospray". Journal of Colloid and Interface Science, 2006, (298), 639-651.

[10] Um, I.C., Fang, D., Hsiao, B.S., Okamoto, A., \& Chu, B. "Electro-Spinning and Electro-Blowing of Hyaluronic Acid”. Biomacromolecules, 2004, (5), 1428-1436.

[11] Stern, R. "Hyaluronan catabolism: a new metabolic pathway", 
European Journal of Cell Biology, 2004, (83), 317-325.

[12] Ji, Y., Ghosh, K., Shu, X.Z., Li, B., Sokolov, J.C., Prestwich, G.D., Clark, R.A.F., \& Rafailovich, M.H. "Electrospun three-dimensional hyaluronic acid nanofibrous scaffolds". Biomaterials, 2006, (27), 3782-3792.

[13] Barrero, A., Gañán-Calvo, A.M., Dávila, J., Palacio, A., \& Gómez-González, E. "Low and high Reynolds number flows inside Taylor cones”. Physical Review E, 1998, (58), 7309.

[14] Hayati, I., Bailey, A.I., \& Tadros, T.F. "Mechanism of stable jet formation in electrohydrodynamic atomization”. Nature, 1986, (319), 41-43.

[15] Hayati, I., Bailey, A.I., \& Tadros, T.F. "Investigations into the mechanisms of electrohydrodynamic spraying of liquids : I. Ef- fect of electric field and the environment on pendant drops and factors affecting the formation of stable jets and atomization". Journal of Colloid and Interface Science, 1987, (117), 205-221.

[16] Sheldon, A.M. "Visualization of fluid motions in Taylor Cones using dye tracer”. Queen Mary, University of London, London. 2009

[17] Barrero, A., Ganan-Calvo, A.M., \& Fernandez-Feria, R. "he role of liquid viscosity and electrical conductivity on the motions inside Taylor cones in E.H.D. spraying of liquids". Journal of Aerosol Science, 1996, (27), S175-S176.

[18] Coleman, P.J., Scott, D., Mason, R.M., \& Levick, J.R. "Role of hyaluronan chain length in buffering interstitial flow across synovium in rabbits”. Journal of Physiology, 2000, (526), 425-434. 